Pediat. Res. 1: 27-38 (1967)

ATP-ase

glucose-6-phosphatase

gluten enteropathy intestine

lactase

leucine aminopeptidase malabsorption

monoamine oxidase

5 -nucleotidase

\title{
Étude histochimique de la muqueuse duodéno-jéjunale dans la maladie coeliaque
}

\author{
J.Jos, J. Frézal, J. Rey et M. Lamy \\ Glinique et Unité de Recherches de Génétique Médicale I.N.s.E.R.M. \\ Hôpital des Enfants-Malades, 149, Rue des Sèvres, Paris XV, France
}

Extract

Eighteen peroral intestinal biopsies were obtained from children with celiac disease. A marked impairment of the enzymatic equipment was noted in the mucosa of patients with untreated celiac disease. The surface epithelium is clearly deficient in ATP-ase, 5-nucleotidase, monoamine oxidase, leucine aminopeptidase, glucose-6-phosphatase and lactase activities. The histochemical reactions for acid phosphatase, non specific esterases, $a$-glucosidases and deshydrogenases are often less affected. Only alkaline phosphatase activity seems unaltered in the surface epithelium.

After the start of a gluten-free diet, an improvement in the patient's clinical condition occurs within a few weeks. At the same time, there is an increase in the activity of some enzymes. The histochemical reaction for ATP-ase shows a rapid improvement. This reaction is almost normal in specimens taken 2, 3 and 5 weeks after gluten withdrawal, but it is still negative in a biopsy performed one week after the removal of gluten from the diet. In addition, the histochemical stains for several other enzymes, such as 5-nucleotidase and leucine aminopeptidase also return towards normal in a short time.

Contrariwise lactase activity remains very low for several months after the beginning of the glutenfree diet. Sometimes the lactase deficiency may be responsible for clinical disturbances, i. e. 'a secondary intolerance to lactose'. In such cases, lactose exerts some 'toxic effect' on the mucosa and prevents the restoration of the villi. Therefore, it is necessary to withdraw lactose as well as gluten from the diet. This intolerance to lactose is nevertheless transient and disappears after the mucosa is repaired.

It should be emphasized that there is a marked discrepancy between the histologic improvement, the evolution of the enzymatic abnormalities and the clinical response after gluten withdrawal. In most cases, the biological and clinical disturbances progressively disappear in three to five weeks. The morphologic changes of the duodeno-jejunal mucosa persist much longer. Before the first two months, there is no evident improvement in the histologic appearance of the mucosa. About three months after the start of dietary treatment, the villi are noticeable again, but are still short and broad (partial villous atrophy). Complete repair may require several months and sometimes more than one year. In striking contrast, some enzymatic activities reappear very quickly. This improvement in some histochemical reactions seems to correlate closely with the rapid clinical response and, therefore, suggests that the histoenzymatic abnormalities play a prominent role in the absorptive failure of celiac disease.

When the patients are put again on a normal diet, histological studies constantly show the reappearance of the mucosal lesions (subtotal villous atrophy), whereas the evolution of both clinical disturbances and histoenzymatic abnormalities is variable. Sometimes a clinical and biological relapse occurs and the histochemical reactions are again impaired. But in most cases, although severe histologic 
changes reappear, the clinical state and the biological tests of intestinal absorption remain satisfactory, with normal growth and no steatorrhoea. It is noteworthy to point out that, in these cases without clinical relapse, the enzymatic activities are only slightly reduced.

\section{Speculation}

Although studies of more cases are needed in order to confirm these data, it appears that histochemical reactions provide a most valuable tool for the study of the mechanism involved in intestinal malabsorption. It seems likely that these reactions reflect the absorptive capacity of the small bowel mucosa. Furthermore, when some patients are put again on a normal diet, the histochemical changes could provide a practical means for predicting a relapse before the impairment of the patient's clinical condition.

Les études histochimiques de la muqueuse intestinale dans la sprue idiopathique de l'adulte [28, 35, 38], ont révélé une diminution de plusieurs activités enzymatiques. Aucune recherche comparable n'a été faite dans la maladie coeliaque de l'enfant.

En revanche, les études histologiques ont montré qu'il existe dans ces deux affections des altérations morphologiques identiques. Les villosités sont élargies, raccourcies, atrophiques. Les cryptes paraissent allongées, l'épithélium superficiel est aplati ou détruit, le chorion est le siège d'une infiltration cellulaire excessive [34]. Ces modifications histopathologiques s'effacent chez l'enfant en quelques mois, lorsqu'on supprime le gluten de l'alimentation, tandis que les troubles cliniques et biologiques s'estompent en quelques semaines [18]. Cette absence de corrélation anatomoclinique est liée pour certains auteurs [1] à une répartition inégale des lésions de l'intestin chez les malades traités. Mais elle peut être expliquée également par des modifications cytochimiques et enzymatiques qui échappent totalement à un simple examen histopathologique. L'histochimie et l'histoenzymologie peuvent à cet égard compléter utilement les investigations habituelles. C'est pourquoi nous avons entrepris une telle étude dans la maladie coliaque de l'enfant. Nous en rapportons ici les résultats.

\section{Matériel}

Nous avons étudié 18 biopsies qui ont été prélevées chez 15 malades (tableau I). Le diagnostic de maladie cœliaque par intolérance au gluten a été porté selon des critères cliniques, radiologiques, biologiques et histologiques qui ont été définis précédemment [17].

Les biopsies ont été recueillies chez trois enfants avant tout traitement (biopsies $\mathrm{N}^{\circ} 1$ a, 2 et 3 ) et dans trois cas au cours d'une rechute (Obs. $N^{\circ} 13,14$ et 15).
Les autres prélèvements biopsiques ont été faits chez des sujets soumis au régime sans gluten depuis une durée variable:

a) tantôt courte: respectivement 1,2,3 et 5 semaines (biopsies $\mathrm{N}^{\circ} 4$ a, 5, 6 et 7);

b) tantôt plus longue:

I. 7 et 10 mois $\left(N^{\circ} 8 a\right.$ et $8 b$ ),

II. un an et plus ( $\mathrm{N}^{\circ} 1 \mathrm{~b}, 4 \mathrm{~b}, 9,10,11$ et 12$)$.

Chez les enfants traités depuis au moins un an, l'examen histopathologique montre une muqueuse intestinale normale ou subnormale. Chez tous les autres, il révèle des lésions duodéno-jéjunales importantes (atrophie partielle sévère ou subtotale).

Dans les cas $\mathrm{N}^{\circ} 1$ a, 2, 3, 4 a et 8, il existait des troubles cliniques et biologiques sévères. Chez les enfants traités respectivement pendant 2, 3 et 5 semaines (Obs. $N^{\circ} 5,6$ et 7) l'amélioration était évidente (disparition de la stéatorrhée et ascension de la courbe de poids). La rémission était complète dans les cas $\mathrm{N}^{\circ} 1 \mathrm{~b}, 4 \mathrm{~b}, 9$, 10,11 et 12, traités depuis un an et plus. Après la réintroduction du gluten dans l'alimentation, deux malades sur trois (Obs. $\mathrm{N}^{\circ} 13$ et 14). conservaient un état clinique et biologique satisfaisant, leur croissance en particulier était normale. Chez le troisième (Obs. $\mathrm{N}^{\circ}$ 15), les manifestations cliniques du syndrome coeliaque étaient réapparues et les épreuves d'absorption étaient à nouveau perturbées.

Dans l'observation $\mathrm{N}^{\circ} 8$, l'intolérance au gluten était accompagnée de troubles cliniques et biologiques évoquant une 'intolérance secondaire au lactose' [33]. Deux biopsies ( $\mathrm{N}^{\circ} 8 \mathrm{a}$ et $8 \mathrm{~b}$ ) ont été prélevées respectivement après 7 et 10 mois de régime sans gluten. Toutes deux révèlent des lésions histopathologiques sévères. L'apport alimentaire en lactose a été supprimé après le second prélèvement biopsique. Cette restriction a amené une disparition rapide de tous les troubles. 
Pour préciser l'histochimie de l'intestin normal, nous avons étudié parallèlement les biopsies de cinq témoins, âgés de 21 mois à 11 ans, choisis parmi des enfants souffrant d'affections diverses, mais sans trouble de l'absorption et sans lésion histologique intestinale (trois colites, une encéphalopathie, une intolérance au lait de vache guérie).

Tableau I. (Seules les biopsies, sur lesquelles ont porté les études histochimiques, figurent sur ce tableau.)

\begin{tabular}{|c|c|c|c|}
\hline Age* & \multicolumn{2}{|c|}{ Régime** } & Histologie $* * *$ \\
\hline $\begin{array}{l}\text { ans/ } \\
\text { mois }\end{array}$ & $\begin{array}{l}\text { sans } \\
\text { Gluten }\end{array}$ & $\begin{array}{l}\text { avec } \\
\text { Gluten } \\
\text { (rechute) }\end{array}$ & \\
\hline
\end{tabular}

\begin{tabular}{|c|c|c|c|}
\hline I.K.F. a & $8 / 4$ & 0 & Atr. Subtotale \\
\hline $\mathrm{b}$ & $9 / 4$ & $1 / 0$ & Normale \\
\hline 2. M.M. & $17 / 5$ & 0 & Atr. subtotale \\
\hline 3. B.M. & $9 / 3$ & 0 & $\begin{array}{l}\text { Atr. partielle } \\
\text { sévère }\end{array}$ \\
\hline 4. B.A. a & $1 / 9$ & 1 semaine & $\begin{array}{l}\text { Atr. partielle } \\
\text { sévère }\end{array}$ \\
\hline b & $2 / 9$ & $1 / 0$ & Subnormale \\
\hline 5. Q.N. & $1 / 5$ & 2 semaines & $\begin{array}{l}\text { Atr. partielle } \\
\text { sévère }\end{array}$ \\
\hline 6. F.M. & $7 / 7$ & 3 semaines & Atr. subtotale \\
\hline 7. B.S. & $0 / 11$ & 5 semaines & Atr. subtotale \\
\hline 8. M.A. a & $2 / 4$ & $0 / 7$ & $\begin{array}{l}\text { Atr. partielle } \\
\text { sévère }\end{array}$ \\
\hline $\mathrm{b}$ & $2 / 7$ & $0 / 10$ & $\begin{array}{l}\text { Atr. partielle } \\
\text { sévère }\end{array}$ \\
\hline 9. B.K. & $8 / 1$ & $1 / 1$ & Subnormale \\
\hline 10. M.E. & $18 / 11$ & $1 / 2$ & Subnormale \\
\hline 11. C.A. & $3 / 5$ & $2 / 0$ & Subnormale \\
\hline 12. C.N. & $2 / 0$ & $1 / 0$ & Subnormale \\
\hline 13. S.A. & $5 / 8$ & $2 / 0$ & Atr. subtotale \\
\hline 14. G.M. & $6 / 1$ & $2 / 0$ & Atr. subtotale \\
\hline 15. L.M. & $4 / 8$ & $2 / 6$ & Atr. subtotale \\
\hline
\end{tabular}

* Age des malades au moment du prélèvement biopsique

** Régimes suivis avant ce prélèvement.

*** Etat morphologique de la muqueuse intestinale.

\section{Méthodes}

\section{Prélèvements histologiques}

Les biopsies ont été prélevées à la jonction duodénojéjunale (angle de Treitz) avec la sonde de BRANDBorg et al. [5] chez les patients à jeun depuis 12 heures. Les fragments ont été immédiatement retirés de la capsule, et congelés, après orientation convenable, à l'aide du dispositif de congélation rapide du cryostat de Harris. Des coupes de $8 \mu$ ont été obtenues à $-18^{\circ}$ dans ce cryostat.

\section{Méthodes histochimiques}

Les réactions suivantes ont été effectuées:

a) Coloration au vert de méthyle-pyronine selon la technique de Brachet [29].

b) Réactions des oxydo-réductases

- Succino-déshydrogénases (NAahlas et al. [22]

- Glucose-6-phosphate-deshydrogénase (WEGMANN et GERZELI [42])

-3-Phosphoglyceraldehyde-deshydrogénase (HimmeLHOCH et KaRNOVSKI [14])

- Deshydrogénase isocitrique (Ogata et Mori [26])

- Deshydrogénase lactique, malique, $\alpha$-glycerophosphorique, $\beta$-hydroxybutyrique et NADH-NADPHtetrazolium-réductases (NAchlas, WalKer et SELIGMAN [23, 24])

- Monoamine-oxydase (Glenner et al. [12]).

L'activité de toutes les oxydo-réductases a été démontrée en utilisant comme accepteur d'électrons le tetranitrobleu de tetrazolium ( $\mathrm{T}$ N B T, Nutritional Biochemical Corp.).

c) Réactions des phosphatases

- Phosphomonoesterases non spécifiques alcaline (Burstone [6]) et acide (Gomori [13], Barka et AnDERSON [3])

- 5-nucléotidase (Wachstern et Meisel [40])

- Glucose-6-phosphatase (GHiQuorne [9])

- Adenosine-triphosphatase (ATP-ase) (méthode de Padykula et Herman [27] modifiée par Wegmann et BANKowski [41])

d) Autres hydrolases

- L'activité aminopeptidasique a été localisée par la réaction de Burstone et Folk [8] en utilisant la Lleucyl- $\beta$-naphtylamide comme substrat.

- Les estérases non spécifiques ont été démontrées par la méthode de Holt [15] modifiée par Pearson et DEFENDi [30] avec le 5-bromo-indoxylacétate.

- La localisation des disaccharidases (maltase, saccharase, tréhalase, palatinase et lactase) a été obtenue par le procédé de DaHLQvist et BRun [10], modifié (J.Jos et al. 1966 [16].

La spécificité des réactions histoenzymatiques a été contrôlée par l'incubation d'une coupe dans le réactif dépourvu de substrat. L'intensité des réactions, dans les coupes étudiées, a été appréciée par comparaison avec une préparation de muqueuse saine, incubée simultanément dans le même godet (Columbia Jar). 

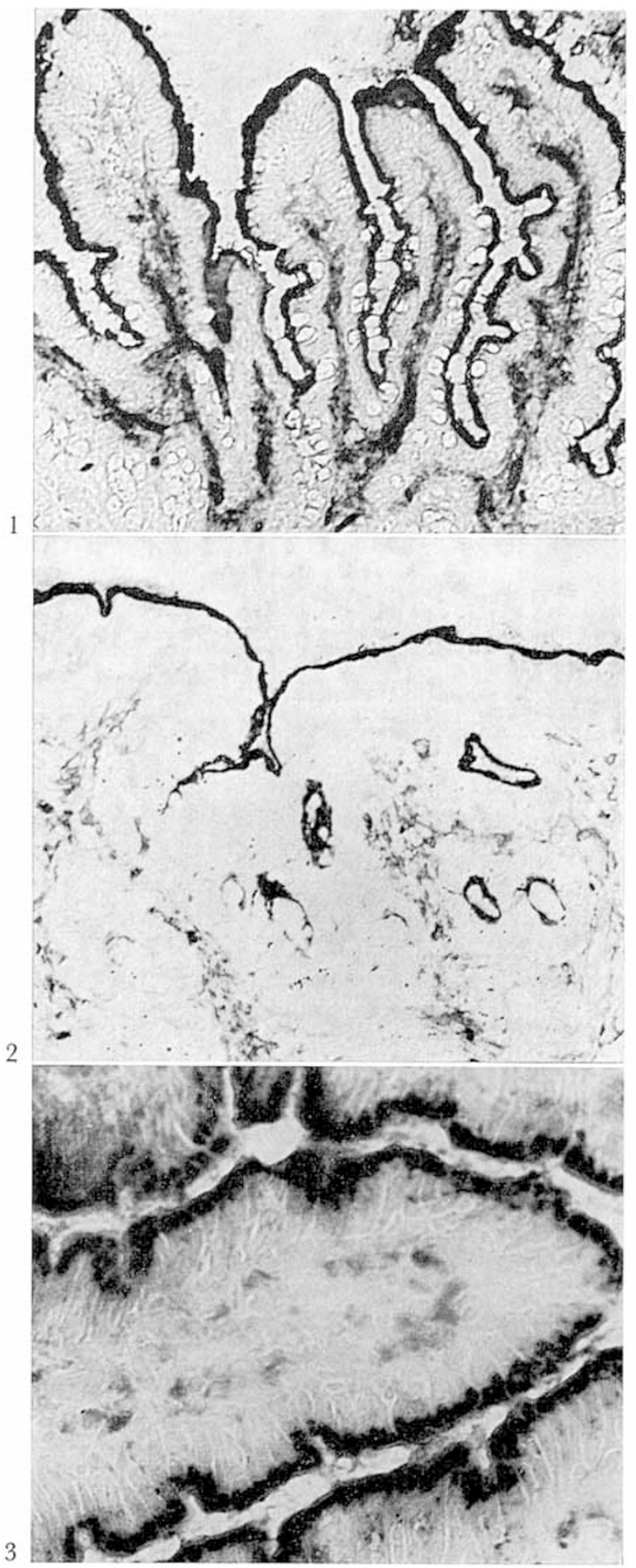

Fig. 1 and 2. Alkaline phosphatase (Burstone method), $(\times 80)$. Fig. 1: Normal intestinal mucosa. Fig.2. Intestinal mucosa in untreated celiac disease; Note the normal activity in the surface epithelium.
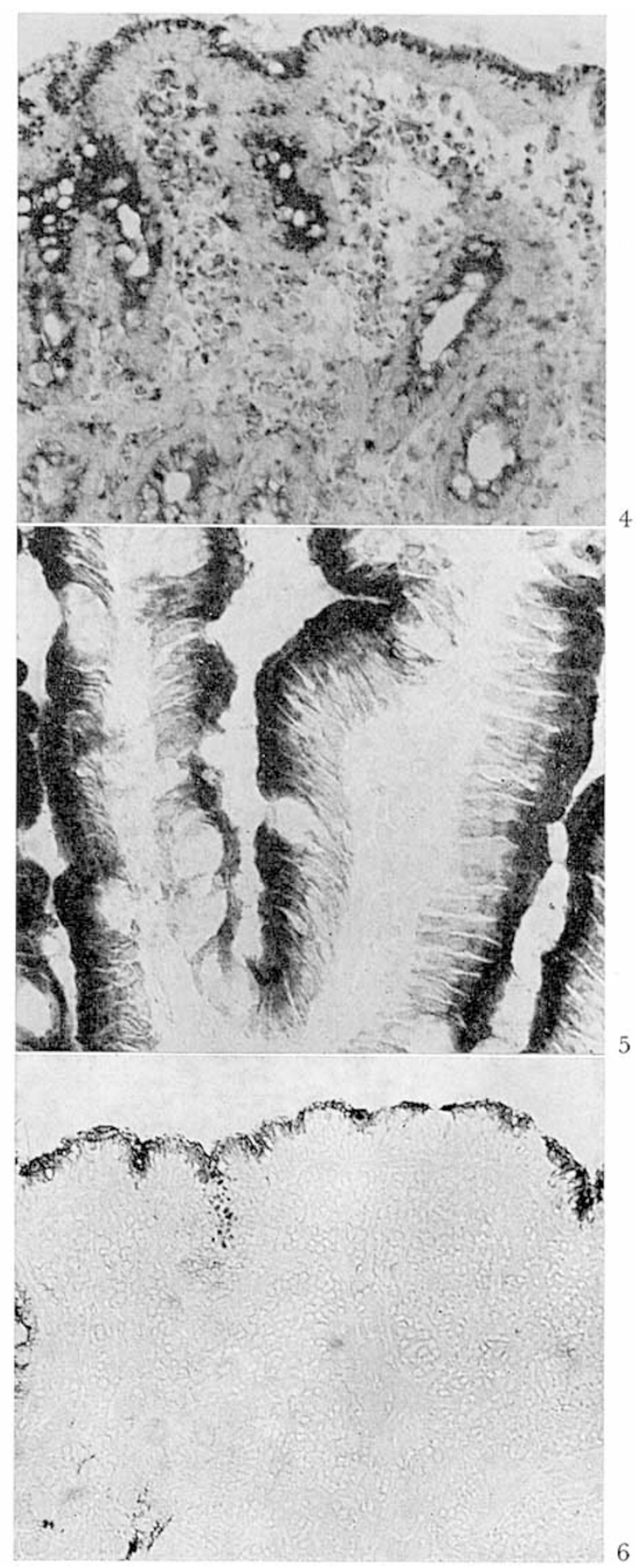

Fig. 3 and 4. Acid phosphatase (Barka and Anderson method). Fig. 3. Normal villus $(\times 250)$. Fig.4. Mucosa in untreated celiac disease $(x 80)$.

Fig.5 and 6. Glucose-6-phosphatase (Chiquoine meth- 


\section{Résultats}

\section{La muqueuse duodéno-jénunale normale}

a) Pyroninophilie. Les cellules épithéliales des cryptes de Lieberkuhn sont fortement colorées en rouge par la pyronine. Cette pyroninophilie diminue progressivement de la base à l'apex des villosités. La pyronine colore également le cytoplasme de quelques cellules chorioniques dont la morphologie rappelle celle des plasmocytes.

b) Les deshydrogénases. La succino-deshydrogénase, les deshydrogénases à NAD et à NADP ainsi que les $\mathrm{NADH}$ et NADPH-tetrazolium-réductases ont une répartition comparable: On note une forte activité dans les hautes cellules qui tapissent les parois des villosités normales et une réaction plus faible dans les cryptes, sauf dans les cellules de Paneth qui sont toujours très actives (fig. 17). En outre les réactions des deshydrogénases à NAD sont légèrement positives dans le chorion, la muscularis mucosae et les vaisseaux de la sous-muqueuse.

c) Les phosphatases. L'activité de la phosphatase alcaline, de l'ATP-ase et de la 5-nucléotidase est intense dans les vaisseaux et la bordure en brosse des cellules épithéliales absorbantes. Ces activités sont absentes dans les cryptes (fig.7). Certaines différences dans la répartition sont caractéristiques: La phosphatase alcaline est localisée dans les capillaires, l'ATP-ase dans le chorion, la 5-nucléotidase dans les vaisseaux de la sous-muqueuse et dans la muscularis mucosae (fig. 12).

Pour la phosphatase acide, il existe trois sites d'activité: le cytoplasme apical des cellules épithéliales superficielles, les cellules de Paneth au fond des cryptes de Lieberkuhn et les macrophages disséminés dans le chorion.

La glucose-6-phosphatase est localisée exclusivement dans le cytoplasme des cellules épithéliales villositaires. Le précipité est plus discret au pôle basal et absent dans le chorion et les cryptes.

d) Estérase non spécifique, aminopeptidase et monoamineoxydase. L'activité estérasique est forte dans l'épithélium des villosités, faible dans les cryptes et absente dans le chorion. Celle de la monoaminemoxydase se répartit d'une façon identique. Celle de l'aminopeptidase au contraire se localise essentiellement dans la bordure des cellules épithéliales. Elle est intense au niveau des

od). Fig.5. Normal activity $(\times 250)$. Fig.6. Enzyme activity in a treated celiac disease, 3 weeks after the start of a gluten-free diet; note the weak activity in the superficial epithelial cells and compare with the reactions of nonspecific phosphatase (fig. 2 and 4$)(\times 80)$. villosités mais diminue rapidement dans la zône des cryptes.

Les disaccharidases. Les $\alpha$-glycosidases (maltase, saccharase, palatinase et tréhalase) sont localisées principalement dans la bordure en brosse des cellules épithéliales qui tapissent les villosités. L'activité lactasique est observée à la fois dans la bordure en brosse et dans le cytoplasme de ces cellules. Il n'y a pas d'activité disaccharasique dans les cryptes [16].

\section{La maladie coeliaque non traitée}

a) Pyroninophilie. Il existe une forte pyroninophilie non seulement dans les cryptes, mais aussi dans l'épithélium superficiel altéré et dans de nombreuses cellules du chorion.

b) Les deshydrogénases. L'activité des deshydrogénases est dans l'ensemble modérément diminuée. Cette réduction affecte principalement l'épithélium superficiel très altéré. Les réactions les plus faibles sont celles de l' $\alpha$-glycérophosphate-deshydrogénase et de la glucose6-phosphate-deshydrogénase. Les mieux conservées sont celles des $\mathrm{NADH}$ et NADPH-tetrazolium-réductases.

c) Les phosphatases. L'activité de la phosphatase alcaline ne paraît guère modifiée dans l'intestin, même très altéré, de maladie cœliaque. En revanche, les réactions de l'ATP-ase et de la 5-nucléotidase sont fortement réduites. Ce double déficit enzymatique semble constant dans la maladie coeliaque non traitée. Il intéresse plus particulièrement la zône superficielle de la muqueuse, mais il peut être global; il en résulte alors une inactivité totale de l'épithélium intestinal. Il contraste avec une réaction souvent très forte de la 5-nucléotidase dans le chorion des cryptes et dans la muscularis mucosae.

L'activité de la phosphatase acide est modérément diminuée dans l'épithélium. Elle est au contraire conservée voire augmentée dans les cellules du chorion.

Il n'en va pas de même pour la glucose-6-phosphatase dont l'activité est toujours considérablement réduite voire absente.

d) Estérase non spécifique, aminopeptidase et monoamineoxydase. L'activité estérasique est nettement diminuée et les réactions de la monoamine-oxydase et de l'aminopeptidase sont souvent très faibles, en particulier dans l'épithélium superficiel.

e) Les disaccharidases. Les réactions histochimiques révèlent un déficit modéré en $\alpha$-glycosidases. En re- 


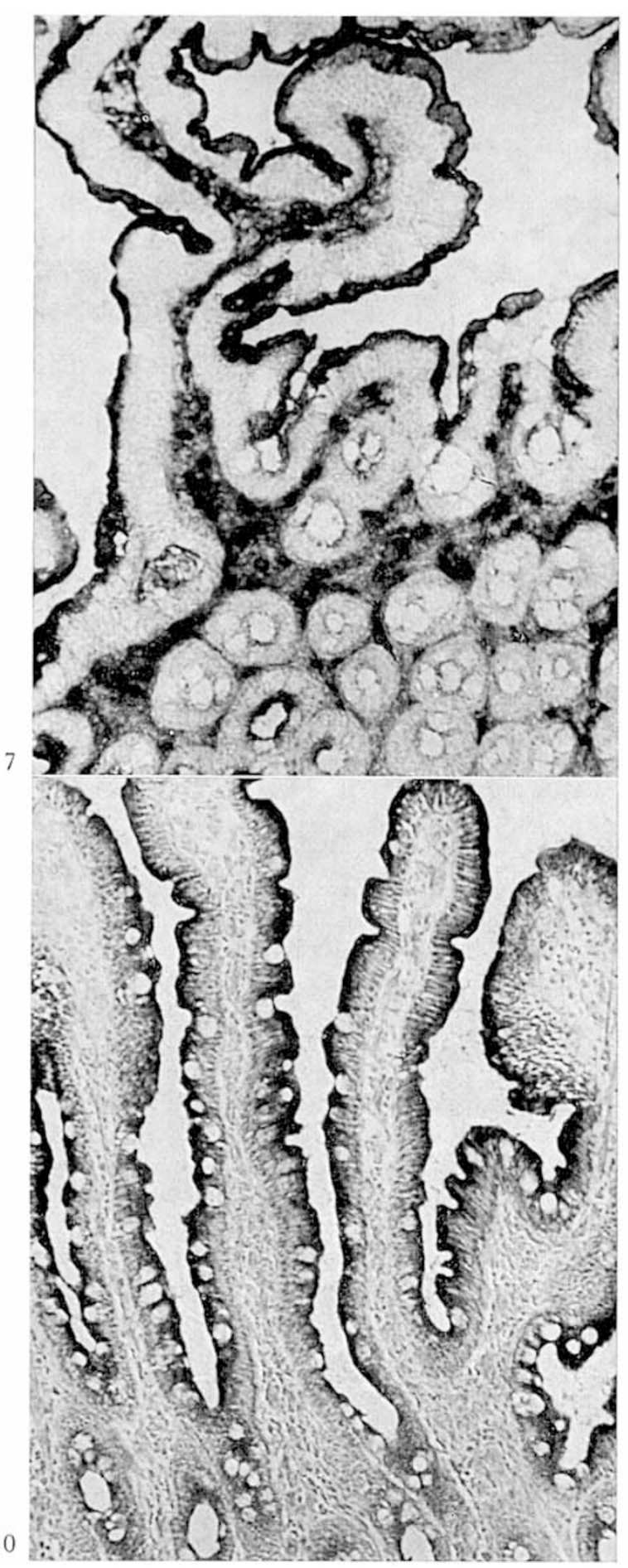

Fig.7, 8 and 9. Adenosine-triphosphatase (at $\mathrm{pH}=$ 9,4). Fig.7. Normal reaction $(\times 250)$. Fig.8. Histochemical staining in untreated celiac disease $(\times 80)$. Fig.9. The same reaction two weeks after the begin-
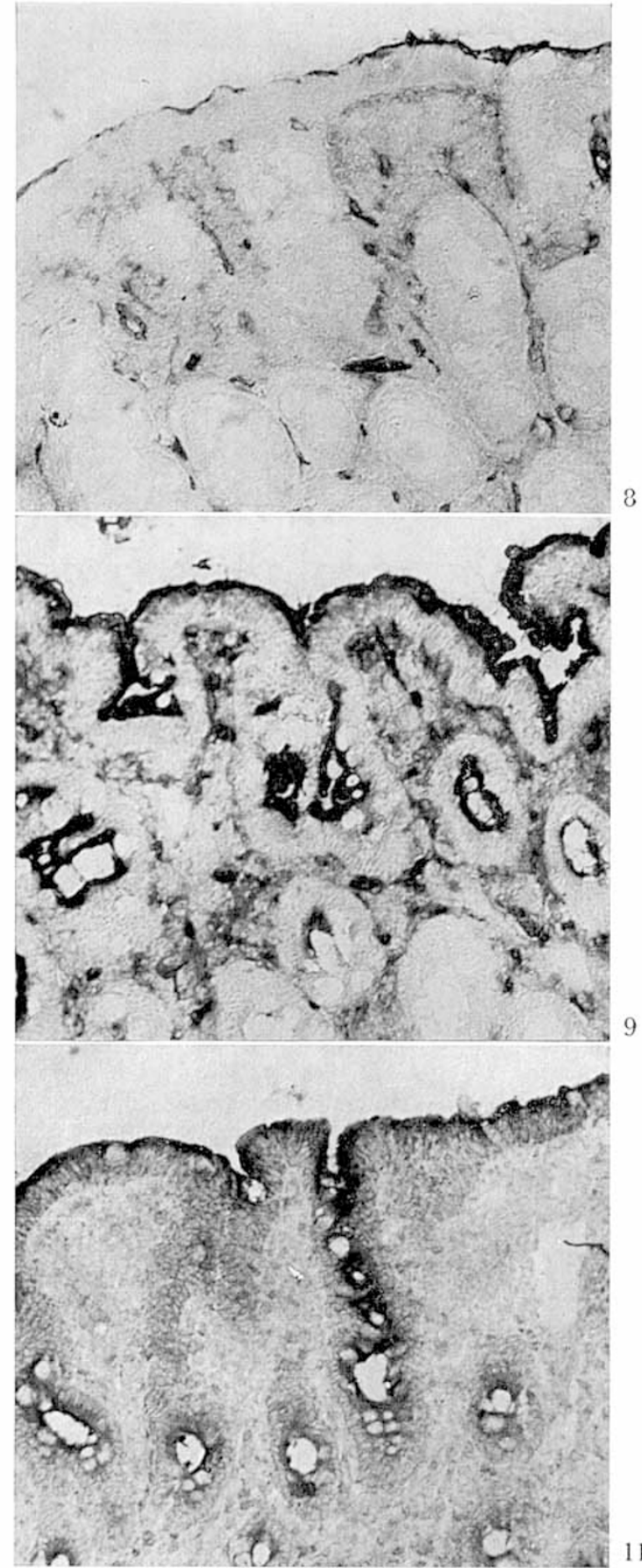

ning of dietary treatment $(\times 80)$.

Fig.10 and 11. Glucose-6-phosphate dehydrogenase $(\times 80)$. Fig. 10. Normal mucosa. Fig.11. Mucosa in untreated celiac disease. 


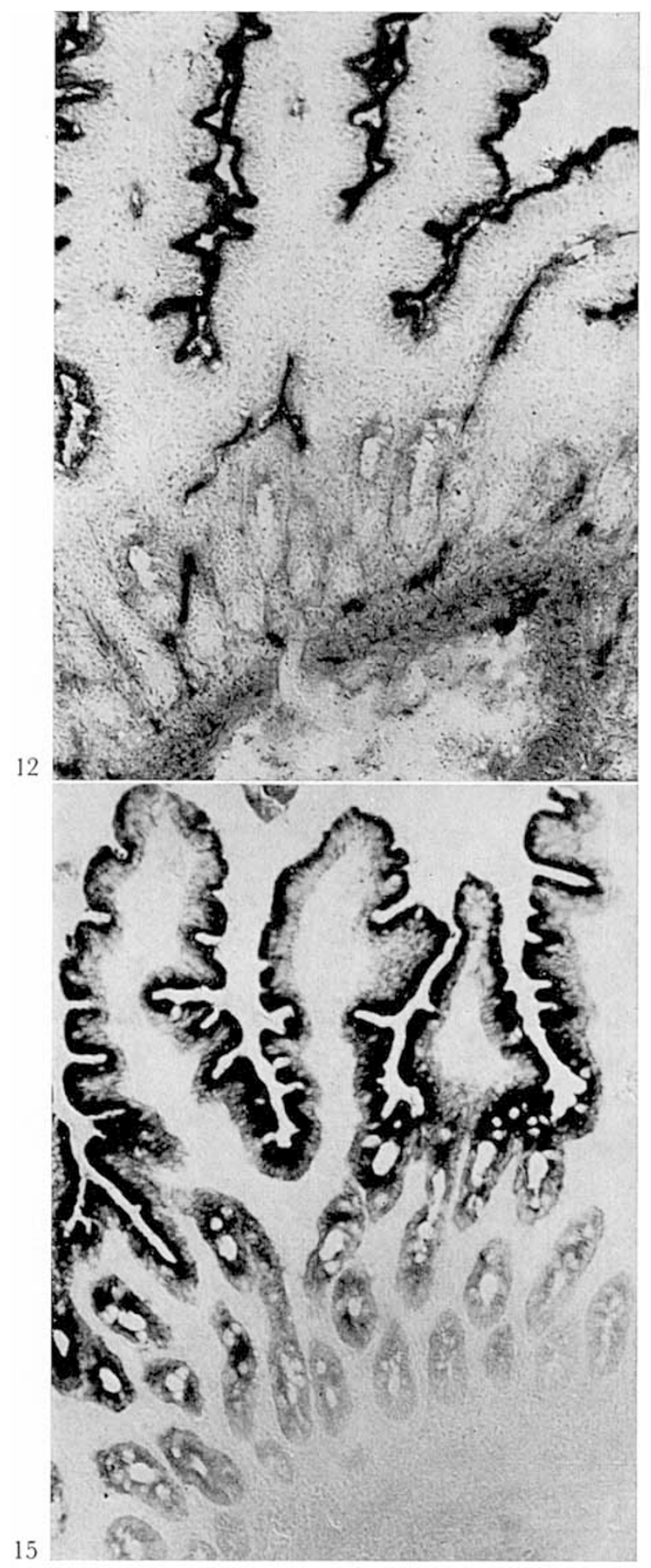

Fig. 12, 13 and 14.5-nucleotidase (Wachstein and Meisel method). Fig. 12. Normal mucosa $(\times 80)$. Fig. 13. Enzyme activity in untreated celiac disease $(\times 40)$. Fig. 14. The same reaction two weeks after gluten withdrawal $(\times 80)$.

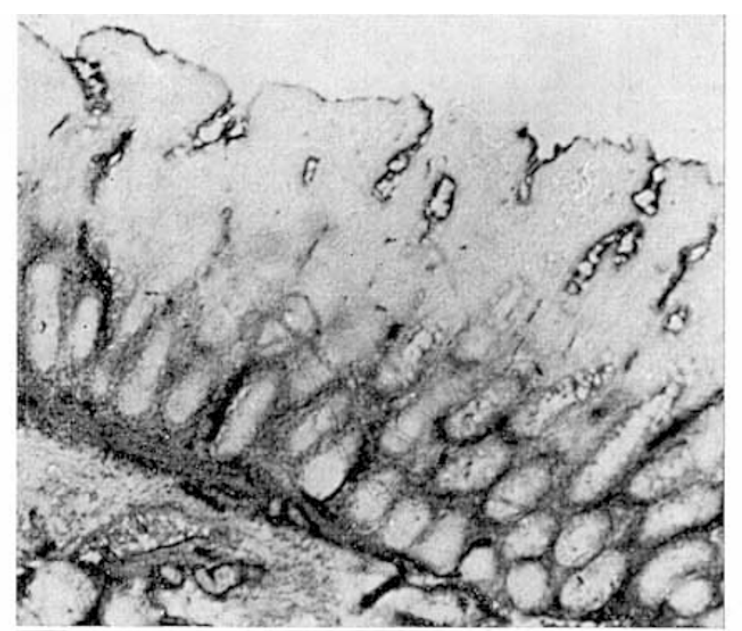

13

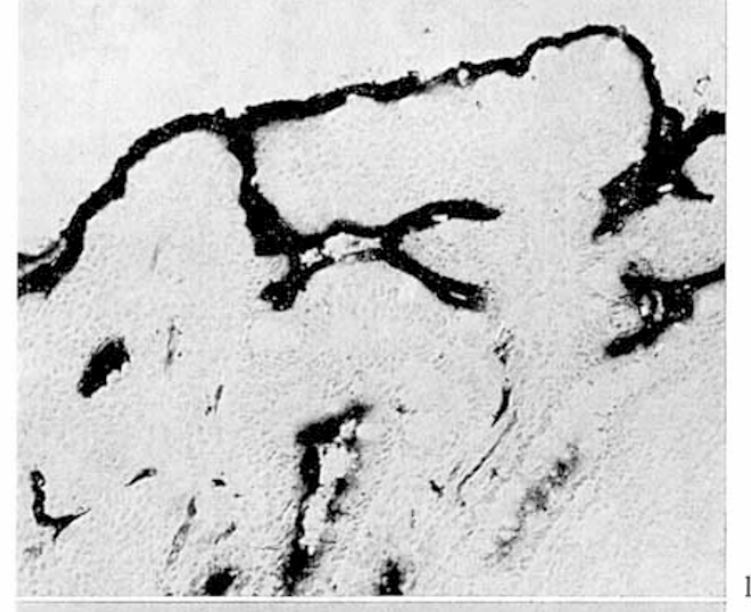

14

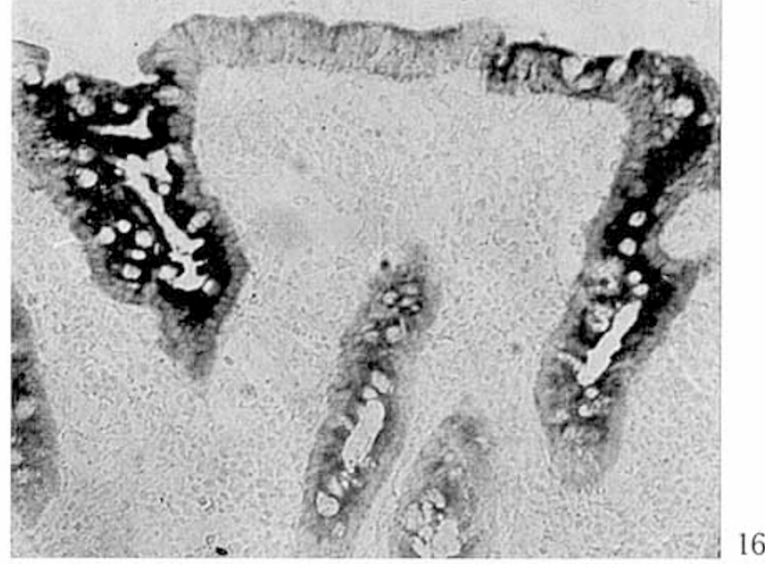

Fig.15 and 16. Non-specific esterase (with 5-bromoindoxyl acetate as substrate) $(\times 80)$. Fig. 15. Normal mucosa. Fig. 16. Mucosa in untreated celiac disease. 


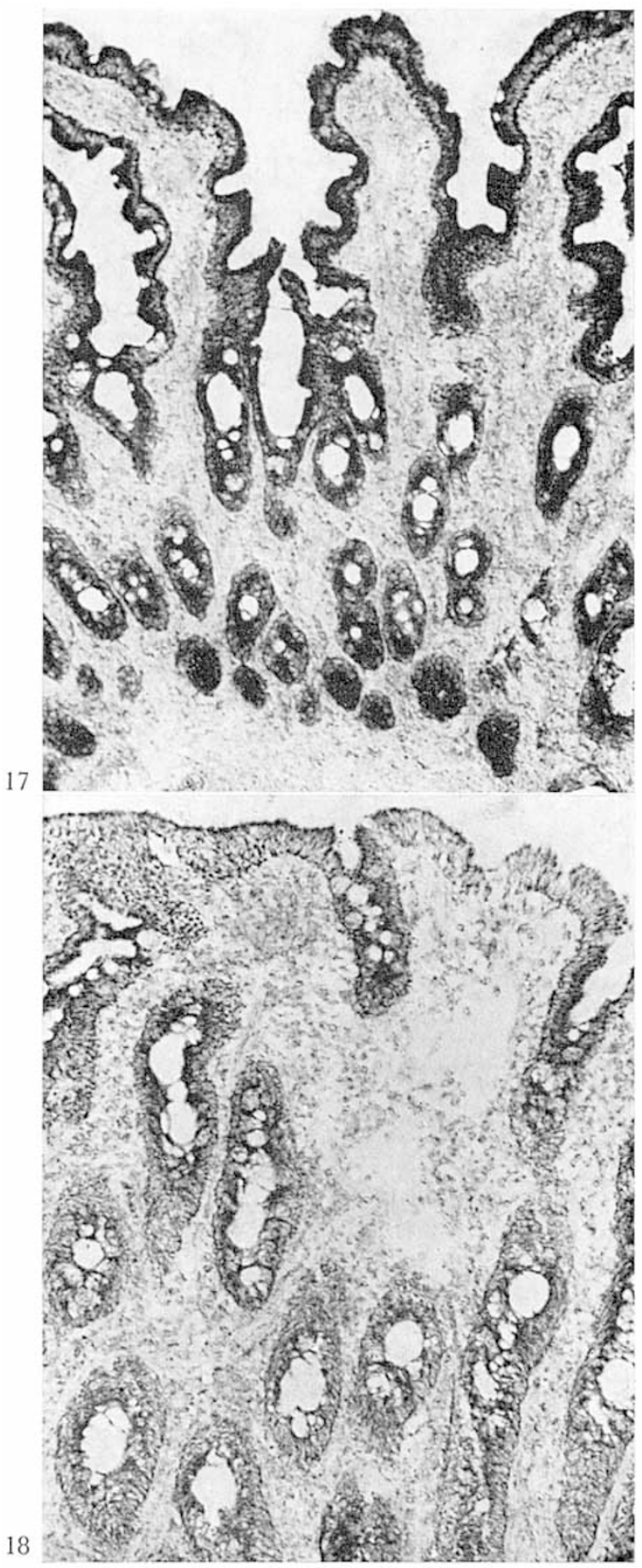

Fig. 17 and 18. Succinic dehydrogenase $(\times 80)$. Fig. 17 . Normal reaction; strong activity is present in Paneth cells. Fig. 18. Enzyme reaction in untreated celiac disease.
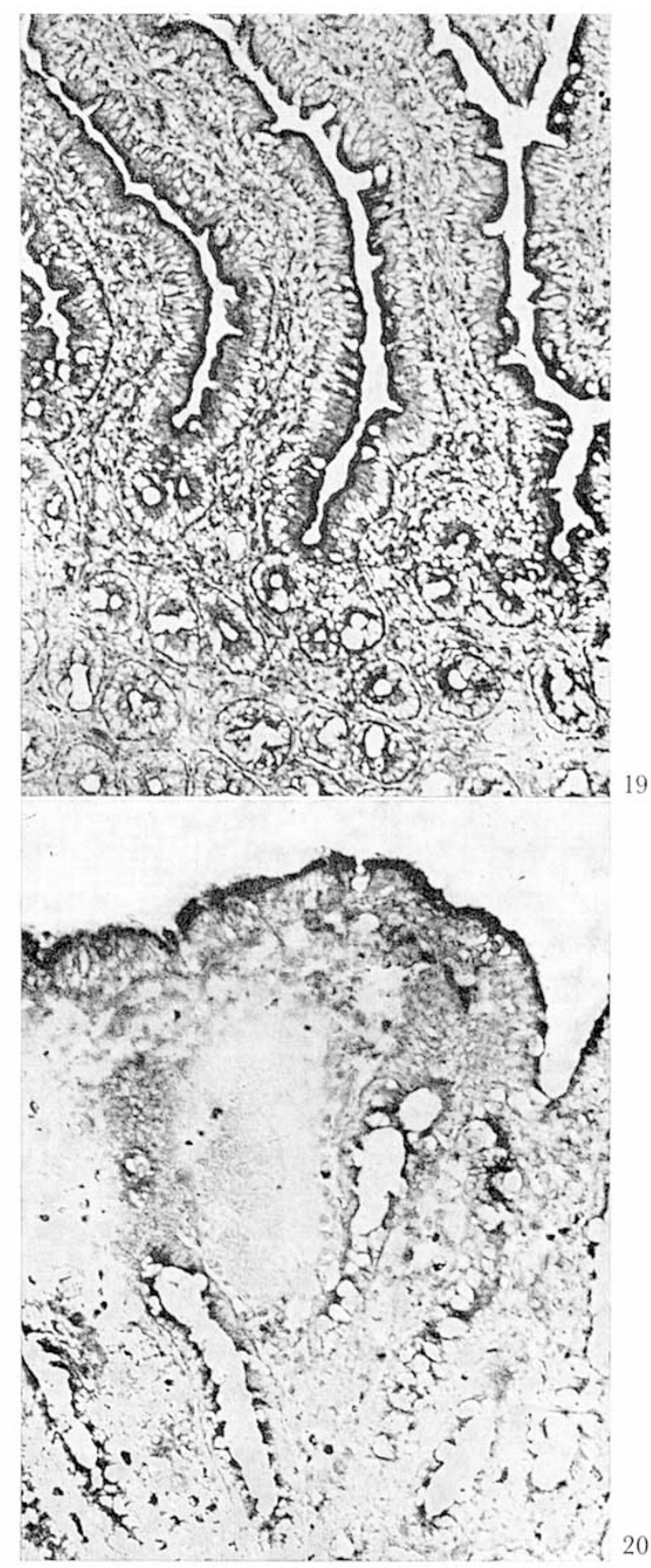

Fig. 19. Normal maltase activity. Note the staining of the brush border $(\times 80)$. Fig. 20. Saccharase activity; untreated celiac disease, duodeno-jejunal mucosa; note the histochemical staining in the surface epithelium $(\times 80)$. 
vanche, l'activité lactasique est souvent considérablement réduite, voire inexistante.

\section{La maladie coliaque traitée}

Les réactions histochimiques se modifient rapidement. Mais la réapparition des activités enzymatiques est plus ou moins précoce selon l'enzyme considérée. Les réactions de l'ATP-ase, de la 5-nucléotidase et de la leucine-aminopeptidase sont d'une intensité satisfaisante dans les biopsies $N^{\circ} 5,6$ et 7, faites respectivement deux, trois et cinq semaines après la mise au régime sans gluten. Au contraire, dans ces mêmes biopsies, d'autres activités enzymatiques sont encore très faibles, en particulier celles de l' $\alpha$-glycerophosphatedeshydrogénase, de la monoamine-oxydase, de la glucose-6-phosphatase et de la lactase. L'activité lactasique est la plus lente à réapparaître. Ainsi, dans les cas traités pendant un an et plus, toutes les réactions, sauf celle de la lactase, sont redevenues normales (Biopsies $\mathrm{N}^{\circ} 1 \mathrm{~b}, 4 \mathrm{~b}, 9,10,11$ et 12 ).

Chez l'enfant dont les troubles cliniques et biologiques évoquaient une intolérance associée au lactose, les deux biopsies ( $\mathrm{N}^{\circ} 8 \mathrm{a}$ et $8 \mathrm{~b}$ ), prélevées respectivement après sept et dix mois de régime sans gluten, montrent une persistance anormale des déficits enzymatiques. Les réactions de l'ATP-ase, de la 5-nucléotidase, de la monoamine-oxydase et de la leucine-aminopeptidase sont en effet très faibles. Celle de la lactase est négative.

\section{La rechute}

Chez les trois malades qui ont été remis à un régime normal après plus de deux ans de traitement (Obs. $\mathrm{N}^{\circ}$ 13,14 et 15) l'examen histopathologique des biopsies met en évidence des altérations très importantes (atrophie subtotale).

Les réactions histochimiques donnent des résultats variables. Dans la biopsie $N^{\circ} 15$, elles sont très faibles. Dans les biopsies $\mathrm{N}^{\circ} 13$ et 14, elles sont moins altérées; les activités de l'ATP-ase, de la phosphatase acide, des deshydrogénases et de la monoamine-oxydase sont certes diminuées, mais le déficit enzymatique reste modéré.

\section{Discussion}

Plusieurs auteurs [4, 21, 28, 35 et 38] ont rapporté des modifications histochimiques intestinales dans les syndromes de malabsorption de l'adulte. Dans la sprue non tropicale, Padykula et al. [28] ont observé une diminution des activités de la succino-deshydrogénase, des estérases non spécifiques, de la phosphatase acide et de I'ATP-ase, qui affecte principalement la zône plate altérée de l'épithélium superficiel. Plus récem- ment, SpIRo et al. [38] ont décrit dans la sprue un déficit enzymatique portant sur plusieurs enzymes (phosphatase acide, glucose-6-phosphate-deshydrogénase, succino-deshydrogénase, NADH et NADPH-tetrazolium-réductases, $\alpha$-glycerophosphate-deshydrogénase, monoamine-oxydase, estérases non spécifiques, $\beta$-glucosaminidase et leucine-aminopeptidase). Notre étude montre qu'il existe dans la maladie cœliaque de l'enfant des anomalies histochimiques comparables. Elle apporte en outre des données complémentaires sur des activités enzymatiques qui n'ont pas été étudiées par d'autres auteurs. De l'ensemble de ces résultats, il paraît ressortir les faits suivants:

1. Dans la maladie coeliaque non traitée, on observe une diminution de nombreuses activités enzymatiques intestinales. Le déficit porte principalement sur les enzymes suivantes: ATP-ase, 5-nucléotidase, glucose-6-phosphatase, monoamine-oxydase, leucineaminopeptidase et lactase. Certaines enzymes paraissent moins affectées: phosphatase acide, estérases non spécifiques, $\alpha$-glucosidase et deshydrogénases. Enfin d'autres gardent une activité apparemment normale; c'est le cas de la phosphatase alcaline.

Les dosages biochimiques de certaines activités enzymatiques donnent des résultats sersiblement différents. Ils montrent en effet une réduction modérée de l'activité phosphatasique alcaline [43,32] et un fort déficit des activités maltasique et saccharasique $[2,37$, 39]. Mais ces différences peuvent être expliquées par la diminution importante de la surface épithéliale dans les muqueuses altérées. Cette anomalie morphologique réduit en effet la proportion des cellules les plus riches en enzymes.

2. La répartition des activités enzymatiques permet de décrire, avec Padykula et al. [28], un certain nombre de zônes:

a) Une zône germinative (zône I) où naissent les cellules épithéliales [19]. Elle est formée par les cryptes de Lieberkühn. Elle est fortement pyroninophile, riche en acide ribonucléique et contient peu d'enzymes. Dans les 'intolérances au gluten', cette zône se situe à la partie inférieure des 'cryptes'.

b) Une zône II qui est le siège d'une faible pyroninophilie et de fortes activités enzymatiques. Cette zône est formée normalement par les cellules absorbantes très différenciées qui tapissent les parois des villosités. Dans la maladie coeliaque, elle est réduite à un court segment épithélial situé à la partie supérieure des «cryptes».

c) Une zône III, pyroninophile et pauvre en enzymes, formée par l'épithélium superficiel altéré. Cette zône est absente dans une muqueuse normale. 
Mais cette division en trois zônes [28] est inconstante et trop schématique. Elle est rarement observée pour certaines réactions histoenzymatiques (glucose6 -phosphatase, phosphatase alcaline, $\alpha$-glycosidases). Au surplus, les activités de la zône II d'une muqueuse altérée, bien qu'elles soient souvent plus fortes, n'atteignent pas l'intensité des réactions villositaires normales.

Le court segment épithélial de la zône II dans les muqueuses de maladie cœliaque est facilement reconnaissable à l'intensité plus forte des réactions à son niveau. Il forme des «pseudo-cryptes» qui sont limitées d'un côté par des ponts intervillositaires et de l'autre par les véritables cryptes de Lieberkühn. Cette disposition est peut-être la conséquence d'une fusion du sommet des villosités. Un tel processus, associé ou non à une «atrophie», pourrait fournir une explication satisfaisante du mécanisme physiopathologique de l'abrasion de la muqueuse.

3. L'étude histochimique chez les malades soumis au traitement met en évidence les faits suivants:

a) L'augmentation des activités enzymatiques est plus ou moins précoce selon l'enzyme considérée. SAMLOFF et al. [35], en étudiant des biopsies itératives, ont observé une réapparition isolée de l'activité ATPasique en quelques jours.

Nos constatations rejoignent celles de ces auteurs. Les réactions de l'ATP-ase retrouvent rapidement une intensité normale. Mais chez nos malades traités, cette amélioration n'est pas aussi précoce. Elle n'est sensible que dans les biopsies prélevées 2 semaines et plus après l'institution du traitement. Elle n'est pas notée dans la biopsie $\mathrm{N}^{\circ} 4 \mathrm{a}$, faite une semaine après la suppression du gluten. En outre, elle n'est pas isolée. D'autres activités enzymatiques, celles de la 5 -nucléotidase et de la leucine-aminopeptidase en particulier, peuvent redevenir normales dans les mêmes délais.

L'évolution des autres activités, et notamment de l'activité lactasique, est bien différente. Celle-ci reste faible ou nulle pendant longtemps. Ce déficit, confirmé par les dosages biochimiques, peut être responsable d'une «intolérance secondaire au lactose» [33]. Dans ce cas, il ne suffit pas d'exclure du régime le gluten, mais il est nécessaire de réduire en outre le lait, au moins pendant les premières semaines du traitement. En effet, le lactose non hydrolysé se comporte apparemment comme un produit toxique et entretient à la fois des troubles cliniques et des lésions de la muqueuse. Notre observation est à cet égard très démonstrative: malgré plusieurs mois de régime sans gluten, les anomalies histologiques et histochimiques persistent chez notre malade sans amélioration appréciable. Cette «intolérance secondaire au lactose» est transitoire et disparaît après la réparation de l'épithélium intestinal
[33]. Des observations analogues ont été rapportées par plusieurs auteurs $[2,25,32,36,37,39,43,44]$.

b) Un autre fait qui ressort de l'étude des malades traités est la dissociation entre l'évolution des lésions histologiques et celle des troubles cliniques, biologiques et histochimiques.

Les troubles cliniques et biologiques s'estompent en trois à cinq semaines [17]. SAmLoff et al. [35] ont observé une amélioration plus précoce encore du transfert cellulaire des graisses dans l'intestin, contemporaine de la réapparition de l'activité ATP-asique.

Les altérations histologiques évoluent plus lentement. Certes, la hauteur des cellules épithéliales superficielles peut augmenter et paraître satisfaisante après quelques semaines de traitement [45], mais I'«atrophie» des villosités persiste longtemps. C'est seulement vers le troisième mois que se dessinent les premières villosités courtes et trapues [18].

La régression en quelques semaines des troubles de l'absorption intestinale s'oppose à la réparation tardive des lésions histopathologiques; elle s'accorde en revanche avec la disparition précoce de certaines anomalies histochimiques. Ces faits paraissent témoigner de l'importance des perturbations histoenzymologiques dans la physiopathologie des troubles de l'absorption.

4. La reprise d'un régime normal, chez les enfants en rémission clinique et histologique, fait renaître les lésions de l'intestin, tandis que l'état clinique et biologique peut demeurer satisfaisant [18]. Il semble que cette meilleure «tolérance» au gluten alimentaire chez certains enfants soit accompagnée d'une conservation relative de l'équipement enzymatique intestinal, comme le suggèrent les observations $\mathrm{N}^{\circ} 13$ et 14 . Mais dans d'autres cas (Obs. $N^{\circ} 15$ ) les troubles cliniques et histoenzymologiques réapparaissent. D'autres études sont cependant nécessaires pour confirmer et préciser ces résultats.

Il convient, en outre, de souligner les risques d'erreurs dans l'interprétation des réactions histochimiques. Il est possible en effet d'observer, sur deux fragments prélevés à des niveaux différents, certaines variations, non seulement dans le degré des lésions [34], mais aussi dans l'intensité des réactions histoenzymatiques [38]. Ainsi, les différences histochimiques entre deux biopsies peuvent être fortuites et résulter de la répartition inégale des altérations. C'est pourquoi nous n'avons retenu dans notre étude que les anomalies importantes, retrouvées à plusieurs examens. D'autre part, l'évaluation des différences dans l'intensité des réactions est délicate sans l'aide de déterminations quantitatives. C'est dire l'intérêt des contrôles biochimiques pour préciser les résultats.

Compte tenu de ces réserves, l'histochimie, à côté des données cliniques, biologiques et histologiques, 
peut être très utile pour juger rapidement des effets d'un régime sur l'intestin. Les réactions histoenzymatiques semblent, en effet, reflèter assez fidèlement l'état fonctionnel et la capacité d'absorption des cellules intestinales.

\section{Conclusion et résumé}

L'étude histochimique de 18 biopsies, prélevées chez des enfants atteints de maladie coeliaque, montre un déficit enzymatique qui porte principalement sur l'ATP-ase, la 5-nucléotidase, la glucose-6-phosphatase, la monoamine-oxydase, la leucine-aminopeptidase et la lactase.

Le régime sans gluten, tout en améliorant l'état clinique des malades, restaure en partie l'équipement enzymatique de l'intestin, avant même la réparation des lésions histologiques. Les premières réactions qui retrouvent une intensité normale sont celles de l'ATPase, de la 5-nucléotidase et de la leucine-aminopeptidase. L'activité lactasique réapparaît tardivement. Ce déficit en lactase est parfois responsable d'une «intolérance secondaire au lactose». Dans ce cas, les lésions de l'intestin sont entretenues par le disaccharide nocif.

Le retour à un régime normal, chez les malades en rémission, fait renaître les altérations morphologiques, tandis que les signes cliniques et les troubles histoenzymatiques peuvent demeurer discrets.

Ces faits mettent l'accent sur l'intérêt des études histochimiques pour élucider les mécanismes intimes des troubles de l'absorption et pour apprécier plus directement les effets du régime sur l'état fonctionnel du revêtement épithélial de l'intestin.

\section{Références}

1. Anderson, C. M.: Histological changes in the duodenal mucosa in coeliac disease: reversibility during treatment with a wheat gluten free diet. Arch. Dis. Child 35: 419-427 (1960).

2. Arthur, A.B.; Clayton, B.E.; Сottom, D.G.; Seakins, J.W.T. and Platt, J.W.: Importance of disaccharide intolerance in the treatment of celiac disease. Lancet r: 172-174 (1966).

3. Barka, T. and Anderson, P.J.: Histochemical methods for acid phosphatase using hexazonium pararosanilin as coupler. J. Histochem. Cytochem. 10: 741-753 (1962).

4. Bolt, R.J.; McCool, S. and Standaert, L.: Histochemical studies of the small bowel mucosa. Acta gastro-ent. belg. 24: 71-75 (1961).

5. BRANDBorg, L.L.; Rubin, C.E. and Quinton,
W.E. : A multipurpose instrument for suction biopsy of the oesophagus, stomach, small bowel and colon. Gastroenterology 37: 1-16 (1959).

6. Burstone, M.S.: Histochemical demonstration of phosphatases in frozen sections with naphtol asphosphates. J. Histochem. Cytochem. 9: 146-153 (1961).

7. Burstone, M.S.: Enzyme histochemistry and its application in the study of neoplasms (Academic Press, New York 1962).

8. Burstone, M.S. and Folk, J.E.: Histochemical demonstration of aminopeptidase. J. Histochem. Cytochem. 4: 217-226 (1956).

9. Chrouorne, D.A.: The distribution of glucose-6phosphatase in the liver and kidney of the mouse. J. Histochem. Cytochem. 1: 429-435 (1953).

10. Dahlevist, A. and Brun, A.: A method for the histochemical demonstration of disaccharidase activities: application to invertase and trehalase in some animal tissues. J. Histochem. Cytochem. 10: 294-302 (1962).

11. Dawson, I. and Pryse-Davies, J.: The distribution of certain enzyme systems in the normal human gastrointestinal tract. Gastroenterology 44: 745760 (1963).

12. Glenner, G.G.; Burtner, H.J. and Brown, G.W., Jr.: The histochemical demonstration of monoamine oxidase activity by tetrazolium salts. J. Histochem. Cytochem. 5: 591-600 (1957).

13. Gomori, G.: Microscopic histochemistry, principles and practice (University of Chicago Press, Chicago 1952).

14. Himmelhoch, S. and Karnovsky, M.J.: The histochemical demonstration of glyceraldehyde-3phosphate dehydrogenase activity. J.biophys. biochem. Cytol. 9: 573-581 (1961).

15. Holt, S.J.: Indigogenic staining methods for esterases; in DANIELLI, J.F.: General cytochemical methods; vol. 1, pp. 375-398 (Academic Press, New York, 1958).

16. Jos, J.; FrézaI, J.; Rey, J. and LAmy, M.: Improvement in the histochemical localization of the intestinal disaccharidases. Application to peroral biopsy specimens. Nature (in press).

17. LAMY, M.; FrezAL, J. et REY, J.: avec la collaboration de Nezelof, C.; Fortier-Beaulieu, M. et Jos, J.: Les stéatorrhées par troubles de l'absorption intestinale; C.R. du XIX ${ }^{\mathrm{e}}$ Congrès de l'Asciation des Pédiatres de Langue Française; p. 161 (Expansion Scientifique Franç., Paris 1963).

18. Lamy, M.; Nezelof, G.; Jos, J.; Frézal, J. et Rey, J.: La biopsie de la muqueuse intestinale chez l'enfant. Premiers résultats d'une étude des syndromes de malabsorption. - Presse méd. 71: 1267-1270 (1963). 
19. Lipkin, M.; Sherlock, P. and Bell, B.: Cell proliferation kinetics in the gastrointestinal tract of man. Gastroenterology, 45: 721-729 (1963).

20. Lodja, Z.: Some remarks concerning the histochemical detection of disaccharidases and gluco-. sidases. Histochemie 5: 339-360 (1965).

21. Myren, J.; Gjone, E. and Skrede, S.: Succinic dehydrogenase activity and histological changes of the small intestinal mucosa in patients with gastro-intestinal diseases. Acta path.microbiol. scand. 64: 161-166 (1965).

22. Naghlas, M.M.; Tsou, K.G.; De Souza, E.; Gheng, C.S. and Seligman, A.M.: Cytochemical demonstration of succinic dehydrogenase by the use of a new p-nitrophenyl substituted ditetrazole. J. Histochem. Gytochem. 5: 420-436 (1957).

23. Naghlas, M.M.; Walker, D.G. and Seligman, A. M.: The histochemical localization of triphosphopyridine nucleotide diaphorase. J.biophys. biochem. Cytol. 4: 467-474 (1958).

24. Naghlas, M.M.; Walker, D.G. and Seligman, A. M.: A histochemical method for the demonstration of diphosphopyridine nucleotide diaphorase. J. biophys. biochem. Cytol. 4: 29-38 (1958).

25. Nordio, S.; La Medica, G. M.; Vignolo, L. and Berio, A.: Lactose intolerance and coeliac disease. Disaccharidases activity in the intestinal mucosa ascertained with the peroral biopsy. Ann. Paediat. 204: 3-23 (1965).

26. Ogata, T. and Mori, M.: Histochemical study of oxidative enzymes in vertebrate muscles. J. Histochem. Gytochem. 12: 171-182 (1964).

27. Padykula, H.A. and Herman, E.: The specificity of the histochemical method for adenosine triphosphatase. J.Histochem. Gytochem. 3: 170-195 (1955).

28. Padykula, H.A.; Strauss, E.W.; Ladman, A.J. and Gardner, F.H.: A morphologic and histochemical analysis of the human jejunal epithelium in nontropical sprue. Gastroenterology 40: 735-765 (1961).

29. Pearse, A. G. E.: Histochemistry; theoretical and applied; 2nd ed. p. 825 (Churchill, London 1960).

30. Pearson, B. and Defendr, V.: A comparison between the histochemical demonstration of nonspecific esterase activity by 5-bromoindoxyl acetate, $\alpha$-naphthyl acetate and naphtol AS acetate. J. Histochem. Gytochem. 5: 72-83 (1957).

31. Plosscowe, R.P.; Berg, G.G. and Segal, H.L.: Enzyme histochemical studies of human gastric and jejunal biopsy specimens in normal and disease states. Amer.J.dig. Dis. 8: 311-318 (1963).

32. Plotkin, G.R. and Isselbacher, K.J.: Secondary disaccharidase deficiency in adult celiac disease (Nontropical sprue) and other malabsorption states. New Engl.J.Med. 271: 1033-1037 (1964).

33. Rey, J.; Frézal, J.; Jos, J. et Lamy, M.: L'intolérance secondaire au lactose. Arch. franç. Pédiat. 23: 488-489 (1966).

34. Rubin, G. E.; Brandborg, L. L.; Phelps, P. C. and TAYLOR, H.G.: Studies of celiac disease. I. The apparent identical and specific nature of the duodenal and proximal jejunal lesion in celiac disease and idiopathic sprue. Gastroenterology 38:28-49(1960).

35. Samloff, M.; Davis, J.S. and Schenk, E.A.: A clinical and histochemical study of celiac disease before and during a glutenfree diet. Gastroenterology 48: 155-172 (1965).

36. Sheery, T.W. and Anderson, P.R.: Disaccharidase activity in normal and diseased small bowel. Lancet $i i$ : 1-5 (1965).

37. Sherling, D.H.; Auricahio, S.; Rubino, A.; Hadorn, B. und Prader, A.: Der sekundäre Mangel an intestinaler Disaccharidase Aktivität bei der Cöliakie. Quantitative Bestimmung der Enzymaktivität und klinische Beurteilung. Helv. paediat. Acta 19: 507-527 (1964).

38. Spiro, H.M.; Filipe, M.I.; Stewart, J.S.; Boorh, G.C. and Pearse, A.G.E.: Functional histochemistry of the small bowel mucosa in malabsorptive syndromes. Gut 5: 145--154 (1964).

39. Townley, R.R.W.; Khaw, K.T. and ShwachMAN, H. : Quantitative assay of disaccharidase activities of small intestinal mucosal biopsy specimens in infancy and childhood. Pediatrics 36: 911-921 (1965).

40. Wachstein, M. and Meiser, E.: Histochemistry of hepatic phosphatases at a physiologic $\mathrm{pH}$. With special reference to the demonstration of bile canaliculi. Amer. J.clin. Path. 27: 13-23 (1957).

41. Wegmann, R. et Bankowskr, Z.: Différenciation histochimique de quatre groupes d'adénosine triphosphatases selon Slater. Ann. Histochim. 5: 121-141 (1960).

42. Wegmann, R. et Gerzelli, G. : La glucose-6-phosphate deshydrogénase et ses corrélations avec des substrats voisins du glucose-6-phosphate. Rôle de l'hexokinase. Ann. Histochim. 6: 111-124 (1961).

43. Welsh, J.D.; Rohrer, G.V.; Drewry, R.; May, J.C. and WAlker, A.: Human intestinal disaccharidase activity. II. Diseases of the small intestine and deficiency states. Arch.intern. Med. 117: 495-503 (1966).

44. Weser, E. and Sleisenger, M.H.: Lactosuria and lactase deficiency in adult celiac disease. Gastroenterology 48: 571-578 (1965).

45. Yardeey, J.H.; Bayless, T.M.; Norton, J.H. and HENDRIx, T.R.: A study of the jejunal epithelium before and after a gluten-free diet. New Engl. J.Med. 267: 1173-1179 (1962). 